\title{
Low-Cost, Risk-Reduction Testing of Class D Spacecraft Photovoltaic Systems
}

\author{
Joshua B Forgione \\ NASA Ames Research Center \\ Naval Air Station, Moffett Field \\ Mountain View, CA 94035 \\ 650-604-0686 \\ Joshua.B.Forgione@nasa.gov \\ Robert Hanel \\ NASA Ames Research Center \\ Naval Air Station, Moffett Field \\ Mountain View, CA 94035 \\ 650-604-5915 \\ Robert.P.Hanel@nasa.gov
}

\author{
Gilbert Kojima \\ NASA Ames Research Center \\ Naval Air Station, Moffett Field \\ Mountain View, CA 94035 \\ 650-604-6503 \\ Gilbert.K.Kojima@nasa.gov \\ Mark Mallinson \\ NASA Ames Research Center \\ Naval Air Station, Moffett Field \\ Mountain View, CA 94035 \\ 650-604-6322 \\ Mark.V.Mallinson@nasa.gov
}

\begin{abstract}
The end-to-end verification of a spacecraft photovoltaic power generation system requires light! A lowcost, portable, and end-to-end photovoltaic-system test appropriate for NASA's new generation of Class D missions is presented. High risk, low-cost, and quick-turn satellites rarely have the resources to execute the traditional approaches from higher-class (A-C) missions. The Class D approach, as demonstrated on the Lunar Atmospheric and Dust Environment Explorer (LADEE), utilizes a portable, metalhalide, theatre lamp for an end-to-end photovoltaic system test. While not as precise and comprehensive as the traditional Large Area Pulsed Solar Simulator (LAPSS) test, the LADEE method leverages minimal resources into an ongoing assessment program that can be applied through numerous stages of the mission. The project takes a true Class D approach in assessing the technical value of a costly, highfidelity performance test versus a simpler approach with less programmatic risk. The resources required are a fraction of that for a LAPSS test, and is easy to repeat due to its portability. Further, the test equipment can be handed down to future projects without building an on-site facility.
\end{abstract}

At the vanguard of Class D missions, the LADEE team frequently wrestled with and challenged the status quo. The philosophy of risk avoidance at all cost, typical to Class A-C missions, simply could not be executed. This innovative and simple testing solution is contextualized to NASA Class D programs and a specific risk encountered during development of the LADEE Electrical Power System (EPS). Selection of the appropriate lamp and safety concerns are discussed, with examples of test results. Combined with the vendor's panellevel data and periodic inspection, the method ensures system integrity from Integration and Test (I\&T) through launch. Following launch, mission operations tools are utilized to assess system performance based on a scant amount of available data.

\section{TABLE OF CONTENTS}

1. INTRODUCTION . .1

2. LADEE OVERVIEW...............................................1

3. LAPSS AND RISK .................................................2

4. MitigATION..........................................................5

5. TEST RESULTS ..................................................8

6. MAINTENANCE, INSPECTION, AND REPAIR....10

U.S. Government work not protected by U.S. copyright
7. MISSION OPERATIONS.........................................10

8. CONCLUSIONS.......................................................11

ACKNOWLEDGEMENTS..........................................11

REFERENCES.......................................................12

BIOGRAPHY ................................................................12

\section{INTRODUCTION}

The Lunar Atmosphere and Dust Environment Explorer (LADEE) is a robotic, lunar-orbiting probe designed to characterize the dust environment near the lunar surface and its atmosphere. [1] The satellite was managed and built at NASA Ames Research Center (ARC). The \$280M, Class D mission (including launch vehicle) began with a successful launch in early September 2013 and will cease its 100-day science operations in spring 2014. The following background on the LADEE satellite and its Electrical Power System (EPS) provides context to a specific project risk and mitigation encountered regarding spacecraft photovoltaic system test.

\section{LADEE OVERVIEW}

\section{The Modular Bus}

Figure 1 shows the completed LADEE satellite in transport during launch-site processing. LADEE is the first spacecraft built on the Ames common bus; an attempt to streamline small satellite development by using a modular 'bus' approach. Each module forms an octagonal ring of the spacecraft, and modules can be expanded or removed as the design requires. The most basic spacecraft configuration consist of a single 'Bus' (B) or 'Single Stage' (S) module. The Bus module is located at the top of the spacecraft, capped by a radiator panel carrying most of the spacecraft avionics. The Bus module is easily recognized by its trapezoidal sections. The $\mathrm{S}$ module, and any additional modules, is built from rectangular sections. The common bus concept is not new within NASA; the strength of the LADEE design is its simplicity and modularity. Initially, 
LADEE only contained three modules. As the spacecraft design matured, it became evident the spacecraft was not long enough to accommodate the entire propulsion system. The LADEE team simply added a module (the 'Extension' (E) module) and moved on with the rest of the mission. Across the four octagonal modules, two rectangular sections are devoted to payloads, and the remaining 30 to the Electrical Power System (EPS) as solar panels.

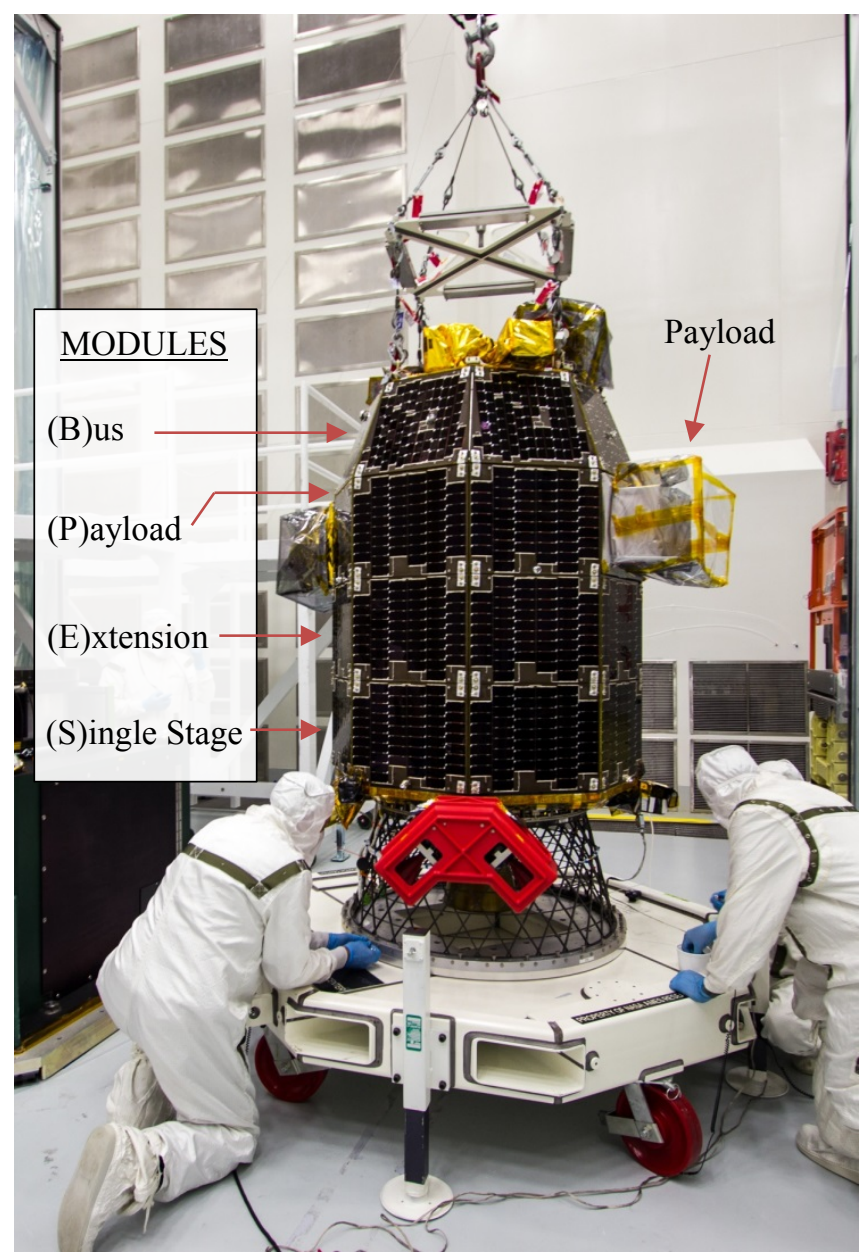

Figure 1: LADEE Spacecraft Hoisted for Encapsulation

\section{LADEE Electrical Power System (EPS)}

Nearly all LADEE observatory hardware is Commercial, off-the-Shelf (COTS) or based on standard products built by aerospace subcontractors. The entire Electrical Power System (EPS), save for the harness, is consistent with this approach. The EPS is a relatively simple, direct energy transfer system consistent with the low-complexity philosophy for Class D missions. [3] No observatory power supply exists. Every payload and avionics unit operates on an unregulated bus voltage that varies with the battery's voltage. Any regulation or isolation is handled internal to each load on the bus. All loads, save for the avionics and communications receiver, are switched on-and-off the bus by a central avionics unit. For power generation, LADEE carries 30 body-mounted solar panels; four octagonal modules' worth minus two sections for payloads. The whole array generates $300 \mathrm{~W}$, nominally. Individual panels generate about 1 ampere in full sun. Sections of photovoltaic power generation system are switched on-andoff the bus as required. Figure 2 illustrates the EPS architecture. Note only a single current-shunt measurement exists for the entire array, and the number of solar array switches (12) is fewer than the available panels. This aspect of the Class D, COTS-driven architecture trickled down through design, assembly, test, and flight-operations.

\section{The LADEE Orbit}

A brief discussion of LADEE's rotation during the lunarorbiting science phase (Figure 3 ) is useful in discussing the design, test, and operation of the power system. In each 113-minute science orbit, the spacecraft completes roughly one full rotation. LADEE's octagonal structure means that, in general, only three sides of the octagon will be illuminated at a time. The body-mounted array is cleverly partitioned into twelve segments (one for each switch), such that a failure on a given switch cannot create a catastrophic failure in generation (Figure 4). The need for partitioning is driven by the COTS circuit card design. The combination of segment-to-switch mapping, direct transfer bus, and nondeployable, non-adjustable arrays translates into the need for an end-to-end EPS test fixture that can easily rotate around the spacecraft, generating light and verifying response.

\section{LAPSS AND RISK}

\section{What is a LAPSS?}

A Large Area Pulsed Solar Simulation (LAPSS) test is generally considered the industry standard for characterizing performance of solar cells, panels, and photovoltaic systems. The test consists of a set of lamps with bulbs matched closely to spectrum of sunlight. In the case of satellite applications, the intensity and spectral emittance is adjusted to approximate that in the space environment. The actual test application is a sequence of light pulses, so as to achieve an impulse response and efficiency of the unit under test. [2] The brief application of light also guarantees little or no thermal stress applied to the solar cells. LAPSS testing can be performed by the panel manufacturer during evaluation, as was the case for the LADEE units. At the observatory level, a LAPSS test can substantially reduce risk by proving the functionality and performance of the power generation subsystem. The array efficiency can be estimated to a level of precision sufficient to detect nonvisible cell defects, critical for long-lifetime arrays. 


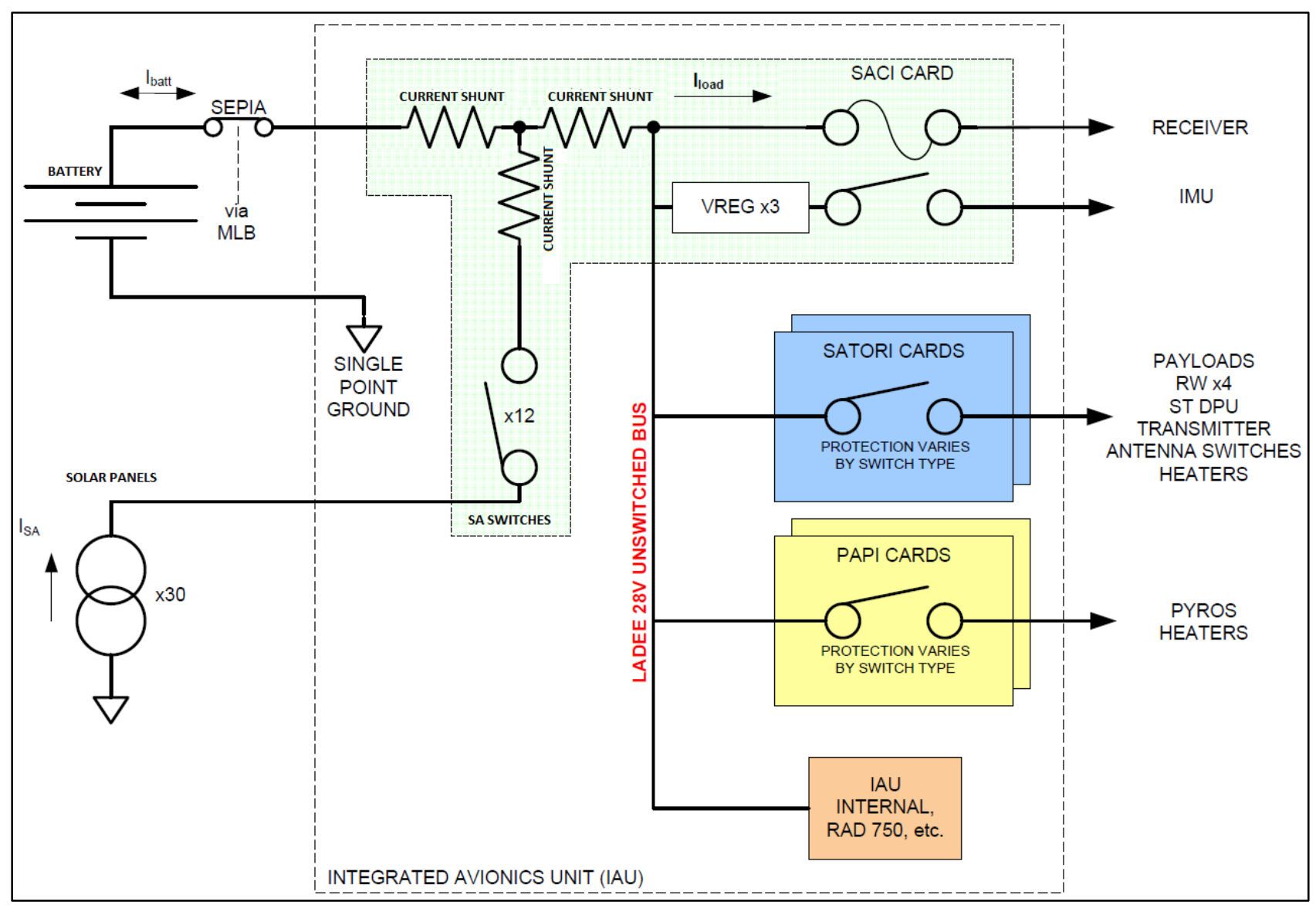

Figure 2: Basic LADEE EPS Block Diagram

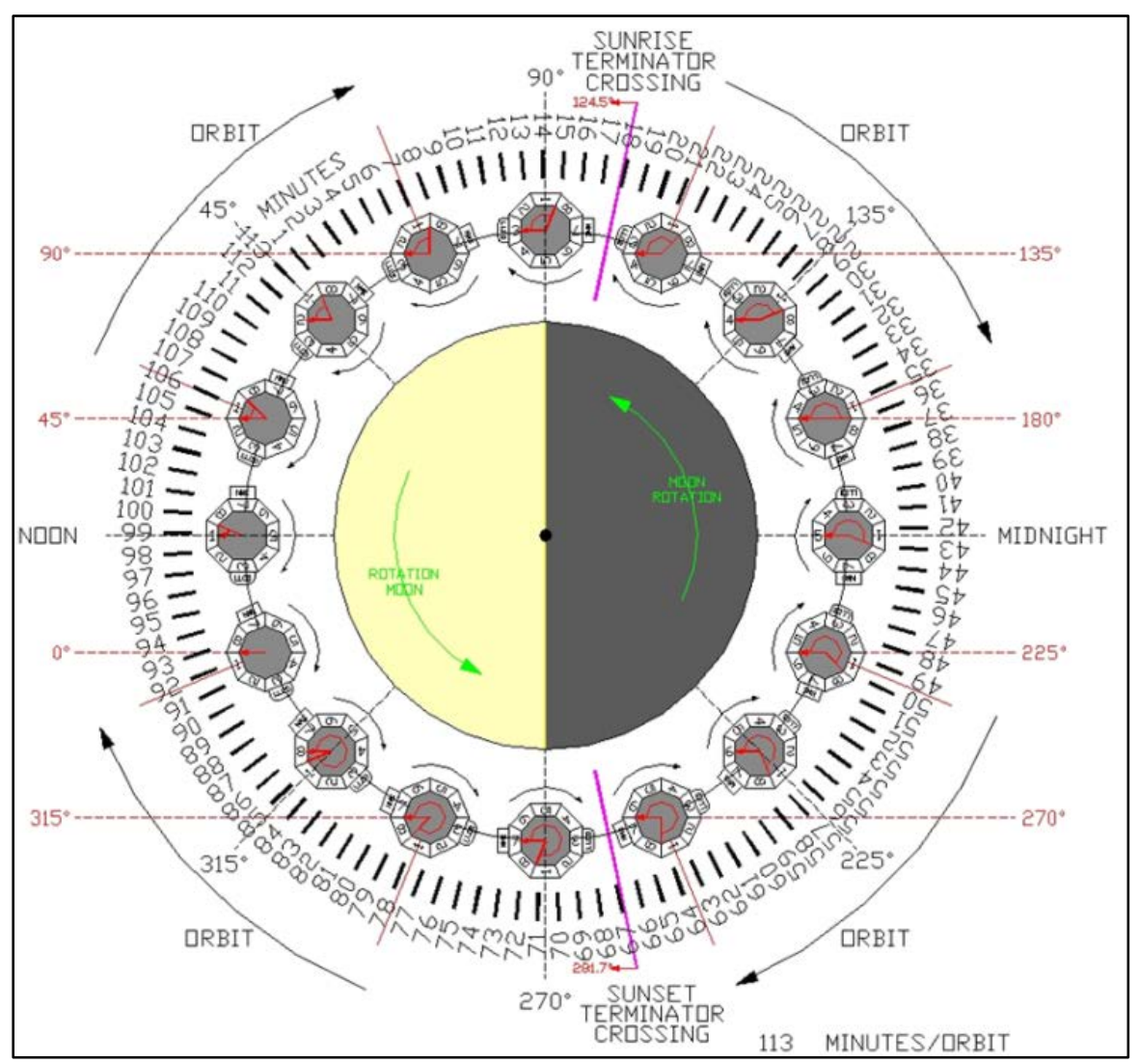

Figure 3: Typical LADEE Science Orbit 


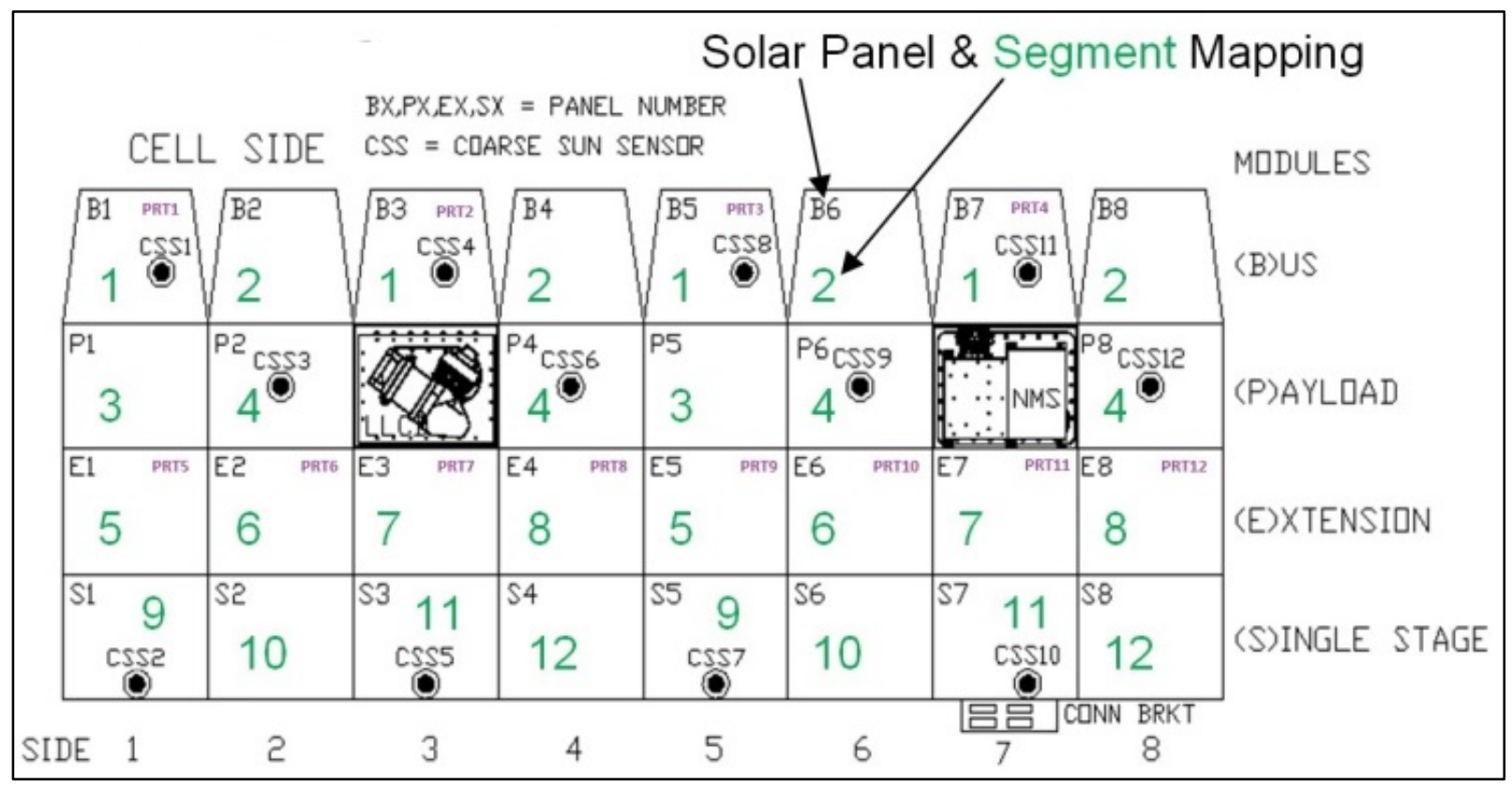

Figure 4: LADEE Solar Panel-to-Switch Mapping Permits Graceful Degradation

\section{LADEE, Risk, and Requirements}

LADEE is part of a new generation of Class D NASA satellites. It is the first satellite of its size fully built at NASA Ames Research Center, which specializes in highrisk satellite development. Commonly referred to as Class $\mathrm{D}$, this risk classification generally applies to programs which are of low cost and complexity, have flexible launch constraints, and short mission lifetimes. [3] This unique type of mission differs from classes $\mathrm{A}$ through $\mathrm{C}$ with respect to acceptable risk, which in turn trickles down into all aspects of system design and test. With respect to photovoltaic system test, Class A-C missions historically utilize the LAPSS test as a standard approach. Higher-complexity power systems, with extended lifetimes or higher consequences of failure, generally require very precise characterization to guarantee long-term performance. LADEE, with its 100-day science phase requirement, can accept a higher risk and did so during integration and test.

The original LADEE environmental test baseline included transfer to another NASA facility with numerous capabilities, including an observatory-quality LAPSS system. Originally, the project held risk \#LADEE-87:

Risk: Given that LAPSS testing will be performed at the vendor, then at observatory-level testing, there is a possibility that late discovery of a solar panel issue will cause a late schedule and cost impact.

Context: Since LAPSS testing facilities do not exist at ARC, there is a concern that panel damage may not be discovered until observatory-level (LAPSS) test.
Initially, the project was rightly concerned about the lack of ability to detect solar panel damage prior to the observatory level LAPSS test. Both risk \#LADEE-87 and the team's risk posture morphed during Phase D. As the project matured, the environmental test baseline changed to a subcontracted approach. [4] The combination of competitive vendors, their cost, available facilities, and diminished schedule reserves resulted in a final selection lacking LAPSS capability. Further, as LADEE entered Phase D, its definitions of risk consequences shifted to accommodate shrinking schedule reserves (Figure 5). Tables 1 and 2 illustrate the change in cost tracking from a percentage of the mission (including launch vehicle) to the cost impact on the observatory alone. These factors combined to redefine and elevate risk \#LADEE-87 from $25^{\text {th }}$ to $3^{\text {rd }}$ in project ranking:

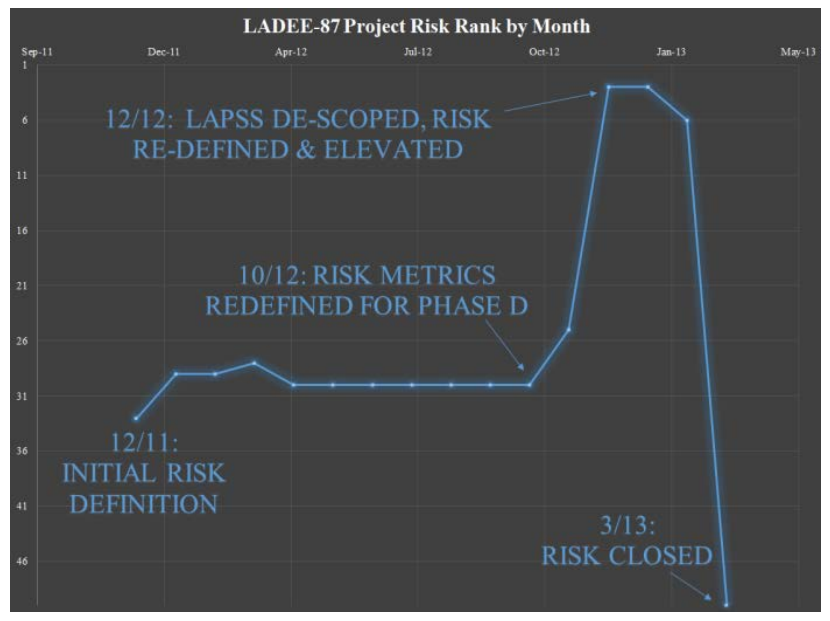

Figure 5: LADEE-87 Risk vs. Time 
Table 1: Original LADEE Risk Definitions

\begin{tabular}{|c|c|c|c|c|}
\hline Rank & Likelihood & Cost & Schedule & Performance \\
\hline 1 & $<1 \%$ & $<0.8 \%$ & Negligible & Negligible \\
\hline 2 & $1 \%$ to $10 \%$ & $0.8 \%$ to $1.5 \%$ & $\leq 1$ months & Minor \\
\hline 3 & $10 \%$ to $33 \%$ & $1.5 \%$ to $2.5 \%$ & 1 to 2 months & Moderate \\
\hline 4 & $33 \%$ to $50 \%$ & $2.5 \%$ to $4 \%$ & 2 to 4 months & Major \\
\hline 5 & $>50 \%$ & $>4 \%$ & $>4$ months & Blocker \\
\hline
\end{tabular}

Table 2: Phase D LADEE Risk Definitions

\begin{tabular}{|c|c|c|c|c|}
\hline Rank & Likelihood & Cost & Schedule & Performance \\
\hline 1 & $<1 \%$ & $\leq \$ 250 \mathrm{k}$ & Negligible & Negligible \\
\hline 2 & $1 \%$ to $10 \%$ & $\$ 250 \mathrm{k}-\$ 500 \mathrm{k}$ & 1 to 2 weeks & Minor \\
\hline 3 & $10 \%$ to $33 \%$ & $\$ 500 \mathrm{k}-\$ 1.0 \mathrm{M}$ & 2 to 4 weeks & Moderate \\
\hline 4 & $33 \%$ to $50 \%$ & $\$ 1.0 \mathrm{M}-\$ 2.0 \mathrm{M}$ & 4 to 8 weeks & Major \\
\hline 5 & $>50 \%$ & $\geq \$ 2.0 \mathrm{M}$ & $>8$ weeks & Blocker \\
\hline
\end{tabular}

Risk: Given that LAPSS testing was only performed for each individual solar panel by the vendor, there is a possibility that without observatory level photovoltaic system testing, the requirements verification of the 295 Watts solar panel output power (EPS-4) will not be verified by test.

The EPS-4 generation requirement text reads as follows:

At a Beta Angle of 0 degrees, after exposure to the space environment in a lunar orbit for 6 months, the minimum total array output including all degradation factors excluding shadowing shall exceed $295 \mathrm{~W}$ at a design voltage of $34 \mathrm{~V}, 80^{\circ} \mathrm{C}$. [5]

\section{Mitigation}

\section{Philosophy}

The LADEE risk tracking approach is not particularly novel, and is fairly standard as compared to other aerospace programs. The innovation is in the risk mitigation. Instead of defaulting to accepting zero risk, LADEE's budget and schedule forced its personnel to consider what is truly necessary to guarantee mission success. The resulting approach is not perfect, but good enough to retire the photovoltaic subsystem technical risk at a massive cost savings. It is the re-evaluation of conventional, risk-averse, and high-cost methods that forms the heart of this work.

\section{Addressing the Risk}

From the power system team's perspective, the risk to requirement \#EPS-4 was secondary to the lack of a proper end-to-end power generation subsystem test. The solarpanel manufacturer already performed LAPSS testing on individual panels; the compiled results of which exceed the EPS-4 requirement. [6] The spirit of the risk was that even though each component of the subsystem passed its respective testing, the system as a whole is greater than the sum of its parts. Specifically, the EPS team cited the following possible causes of system-level failure:

- Incorrect or swapped solar panel connectors. Will manifest as mis-mapping panels to solar-array switches.

- Damage or degradation to the COTS PCB carrying the solar array switches.

- Proper harness connections, but corrosion or degradation leading to an increased harness resistance. Would only manifest at higher currents and lead to a power loss or shift in the panel's current 'knee.'

- An individual string within a panel is degraded, damaged, or failed following delivery and installation.

- A software bug in the code driving the solar array switches. Will manifest as closing a switch to the wrong solar array segment.

- Improper connections or software for measuring the PRT temperature sensors embedded in selected solar panels.

- Improper connections or software for measuring the Coarse Sun Sensors embedded in selected solar panels.

The standard LAPSS test method, appropriate to Class C and higher missions, would expose any and all of the above failure modes. NASA ARC does not have an on-site facility, and the funding $(\sim \$ 100 \mathrm{k})$ and months to construct and calibrate were simply not available. Even if it were available, a singular LAPSS test has limitations of its own. Subsequent panel damage due to handling may not be revealed until post-launch. The two months to ship the 
spacecraft and perform the test are costly and risk-prone. The EPS team therefore needed a low-cost, quick-turn approach. A review of the detailed risks reveals none is particularly sophisticated or necessitating high technology. Most are related to functionality only, and the performance requirements do not imply that efficiency characterization is even necessary. Therefore, a LAPSS facility would be overkill. The team simplified the process by making a key decision:

- Formally verify requirement \#EPS-4 using the manufacturer's per-panel LAPSS test data.

- The test results are consistent across each panel type, and will also be used for system modeling and initial mission operations allocations.

The general process for an end-to-end test can now be boiled down to the following basic flow: [7]

1. Set up a lamp aligned with the center of a single panel at a safe distance from the panel.

2. Configure the avionics' software to open all solar array switches, save the switch and segment associated with the panel under test.

3. Warm up the lamp (covered) for one minute (nominal)

4. Quickly remove the cover, and record the spacecraft solar array current.

5. Continue to illuminate the spacecraft, monitoring current and temperature until the system stabilizes. Abort if safety limits are violated.

6. Power off the lamp.

7. Move the lamp to the next panel and repeat the process. Maintain the same lamp configuration and distance for consistency.

\section{Selecting and Tuning the Lamp}

Picking the lamp system was largely driven by the type of bulb required; it and subsequent tuning required the most engineering effort in the activity. Initially, the LADEE team used a small, handheld halogen lamp. Unfortunately, the panels only generated about $30 \mathrm{~mA}$ using this light, or $3 \%$ of their rating. The initial impulse was to simply find a higher wattage lamp. On further investigation, the spectrum generated by halogen is not the best solution for testing solar panels. Sunlight, especially without atmospheric attenuation or distortion, can be approximated by Planck's blackbody equation. [7] Any man-made bulb will differ from the ideal sunlight spectrum to some degree; however, a halogen bulb will tend to generate less UV and more infrared than sunlight. This translates into less energy transformed to current, and more energy manifested as heat. Therefore, a very high power halogen lamp is required to generate current close to a panel's rating, at the risk of overheating the panels. That said, halogen is attractive as a solution in that many inexpensive and portable COTS options are available. Further research into theatre lighting revealed metal-halide lamp systems as another option. Specifically, metal-halide bulbs are typically used in highpower theatre lamps sold as 'sunlight' lamps. These lamps are designed to simulate sunlight for indoor filming or photography.

Fortunately, lamps of this type are easily rented by theatre supply houses. The EPS team rented a couple lamps at a relatively inexpensive rate $(\sim 750$ per week) and ran experiments on a flight qualification panel (Figure 7). Relatively few NASA engineers are theatre lighting operators; the qualification (qual) panel was ideal for this experiment. The qual panel is a factory-tested unit from the same lot as the flight panels; any results translate easily to the spacecraft test. Being a non-flight spare panel, testing can occur outside of a cleanroom environment, with plenty of space and no major concerns regarding damage or degradation. The team built a panel test fixture and mounted the whole assembly to an optical bench. Connections were provided measuring current and embedded-sensor temperature (Figure 7). Testing involved iterative adjustment of the lamp distance, focus, and on-time to find a combination of single-panel spot size and sufficient current, at a safe temperature. Table 3 shows the parameters the team chose for a safe system test: [7]

Table 3: Lamp Configuration Parameters

\begin{tabular}{|l|l|}
\hline Parameter & Value for Flight Test \\
\hline Height & $\begin{array}{l}\text { Varies based on spacecraft orientation. } \\
\text { Align with panel center. }\end{array}$ \\
\hline Distance to Panel & No closer than 36 inches. \\
\hline Lamp Focus & $\begin{array}{l}\text { Roughly 15\% spot for 25\% de-rated } \\
\text { current with a spot size of one panel. }\end{array}$ \\
\hline $\begin{array}{l}\text { Illumination } \\
\text { Time }\end{array}$ & $\begin{array}{l}1 \text { minute warm-up with scrim cover } \\
\text { installed, followed by up to } 3 \text { minutes } \\
\text { with scrim removed }\end{array}$ \\
\hline
\end{tabular}

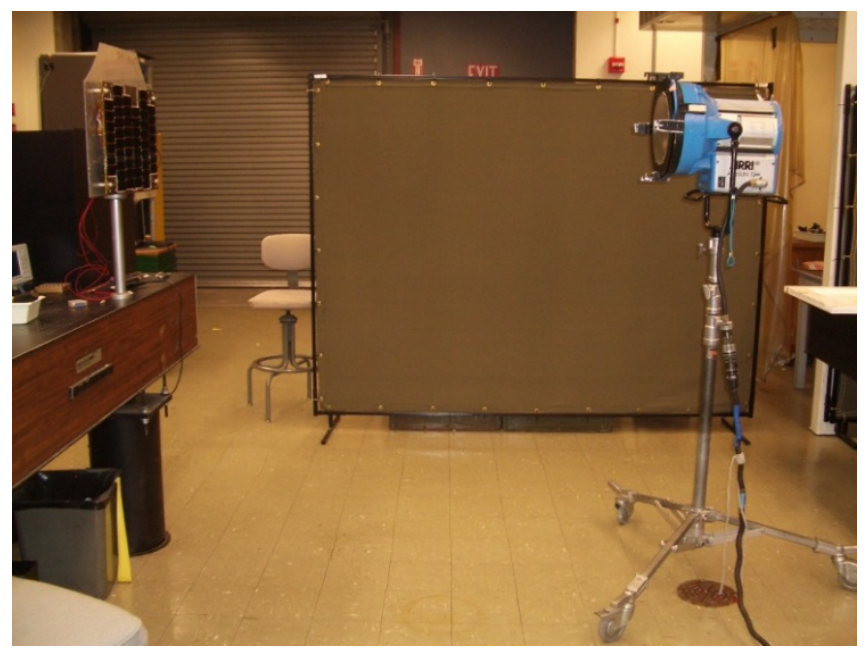

Figure 7: Experimenting with the Qualification Panel 
The team settled on an $1800 \mathrm{~W}$, Hydrargyrum Medium-arc Iodide (HMI; i.e. metal-halide) lamp system, with ballast and adjustable stand. Figure 8 shows the scaled lamp spectrum over-laid onto a $6000 \mathrm{~K}$ blackbody spectrum. [8][9] The total purchase price for the system is $\$ 11 \mathrm{k}$ with a three-week lead time.

\section{Selecting the Current:}

The amount of current required for a 'passing' test was the matter of some debate. Ideally, the maximum panel current would be generated so as to meet the EPS-4 requirement by test. However, the theatre lamp is not that precise; slight variations in warm-up time, distance, and focus can produce large swings in panel current. The lab experiments also showed that some cases can generate currents in excess of the design value. To eliminate risk of hardware damage, the flight test requires a minimum of $500 \mathrm{~mA}$ generation, and a target of $80 \%$ of the manufacturer's LAPSS measurement. [7] This value is large enough to expose any substantial power losses in signal path, and to cause a failed string to clearly result in an out-of-family measurement.

\section{Hardware and Personnel Safety:}

Thermal-In addition to safely de-rating the current generation, several safety aspects required resolution prior to the first flight test. Though the metal-halide bulbs better approximate the sunlight spectrum, they still generate infrared. Both the panel and surrounding flight hardware require real-time monitoring to prevent thermal-related damage. Only $40 \%$ of the LADEE panels carry embedded temperature sensors. To maintain hardware safety, the test requires use of a calibrated, handheld thermal imager. The specific imager used (Fluke Ti25) is essentially an infrared digital camera. The entire panel can be monitored and saved for offline analysis. The test limit for panel temperature is $70^{\circ}$ Celsius, driven by the mounting bushings. This proved good for nominal safety measurements, but limited for precision use due to surface reflections.

Contamination-LADEE is a contamination-sensitive mission due to the presence of mass-spectrometers and optical instruments. All spacecraft activities occur in $10 \mathrm{k}$ clean tent, and instruments are continuously bagged and purged. Theatre lamp systems are not necessarily designed for a clean-room environment. The rental lamps see heavy usage, and often have peeling paint. New lamps outgas substantially, and must be burned in prior to test in the clean tent.

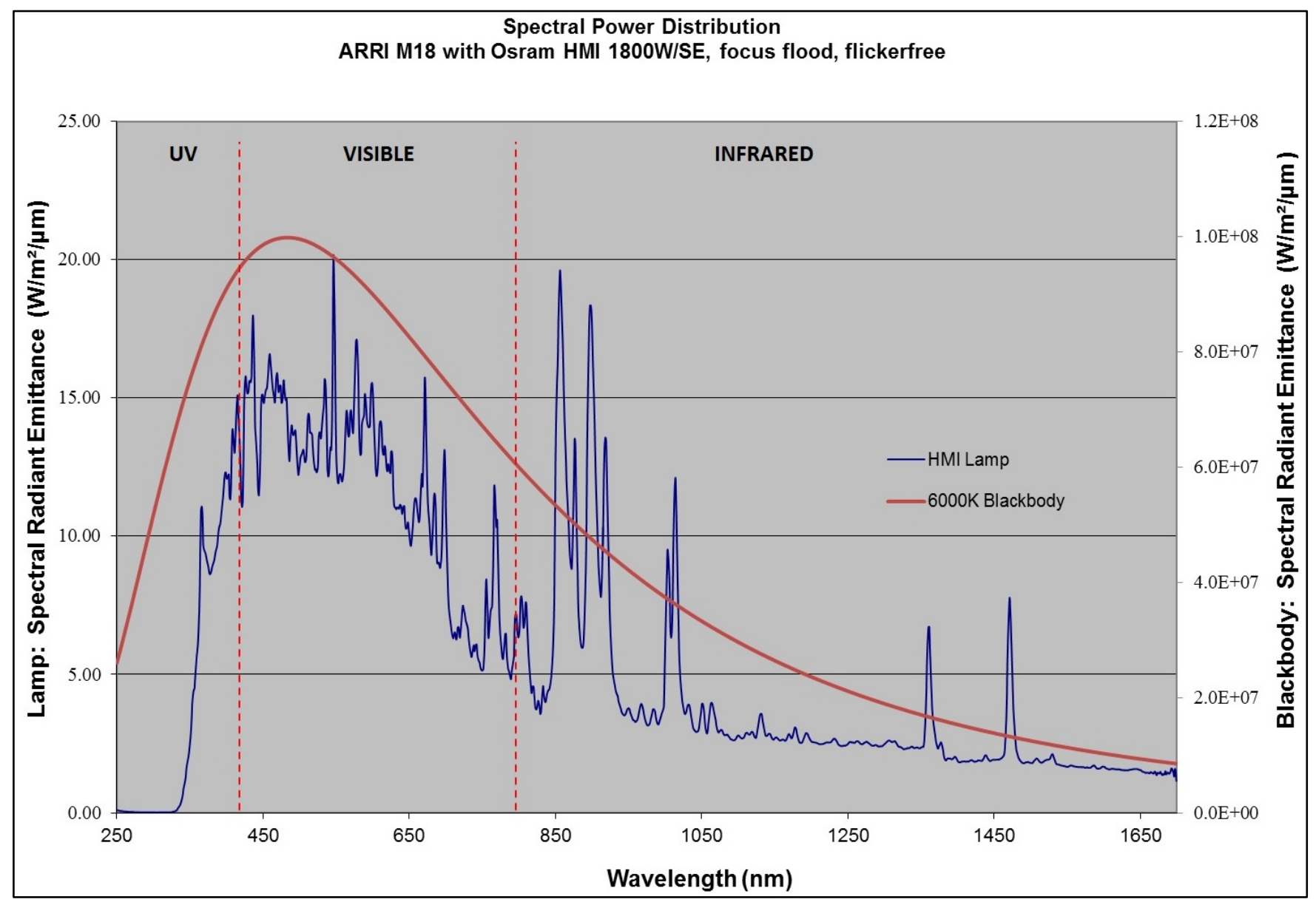

Figure 8: Test Lamp Spectrum 
Optics - Though the lamp is, roughly-speaking, meant to simulate sunlight spectrum and intensity, most of the optical hardware on the spacecraft is not designed to look directly into the sun. LADEE's body mount panels are right next to the payloads; for protection, all instruments were shielded with reflective blankets. LADEE's star trackers can withstand sunlight, and did not require protection.

Personnel Safety-Regarding personnel safety, precautions were necessary. One of the strengths of the metal-halide bulb is less infrared and more UV; unfortunately human eyes are sensitive to UV radiation. Further, the light reflects easily and can cause headaches even when personnel avoid looking at the bulb. Fortunately, an inexpensive, stock solution was available. Most standard safety glasses meet the ANSI Z87.1 standard for UV protection. Welding glasses also meet the standard, and often provide fitted, wrap-around protection. To meet personnel safety, the project purchased a dozen glasses and required all personnel to wear them during test. [7] Debriefs and placards serve to notify all staff.

\section{Test Results}

The first test occurred just after final observatory integration, and was straightforward to execute. The test time was about four hours, and required three staff to operate. The EPS-4 295W requirement was not met due to de-rating the lamp. Figures 9 and 10 are time series of all panel currents and temperature sensors during the test.
Figure 11 shows a typical 'step' current signature for each panel type. Note the signature shows evidence that the lamp warm-up persists beyond one minute. All panels measured above the $0.5 \mathrm{~A}$ requirement, with most between $70-75 \%$ of maximum. Typical panel temperatures hovered around $45^{\circ}$ Celsius (Figure 12), with the hottest at about $59^{\circ}$ Celsius. The test was considered a success, and LADEE Risk \#87 was subsequently closed.

LADEE project management was sufficiently happy with the test that it approved purchase of a new lamp system. The test proved to be simple enough in execution that the test rig could be taken to the launch site for evaluation prior to encapsulation. At the final observatory test (Figure 13), the team attempted verification of the $295 \mathrm{~W}$ requirement. Enough thermal margin was available to relax the generated current de-rating from $25 \%$ to roughly $10 \%$. Recall that only one panel can be tested at a time; analysis combines superposition of results with the spacecraft rotation and orientation outlined in Figure 3. The average observatory power generation meets the $295 \mathrm{~W}$ requirement (Table 4). Three sides of the spacecraft fell less than $10 \%$ short of the requirement. However, the level of residual risk was considered appropriate for a Class D mission and no further testing was required.

Table 4: EPS-4 Closed by Test

\begin{tabular}{|l|l|l|l|}
\hline $\begin{array}{l}\text { Minimum } \\
\text { Power }\end{array}$ & $\begin{array}{l}\text { Maximum } \\
\text { Power }\end{array}$ & $\begin{array}{l}\text { Average } \\
\text { Power }\end{array}$ & Requirement \\
\hline $270.9 \mathrm{~W}$ & $339.7 \mathrm{~W}$ & $310.4 \mathrm{~W}$ & $295 \mathrm{~W}$ \\
\hline
\end{tabular}

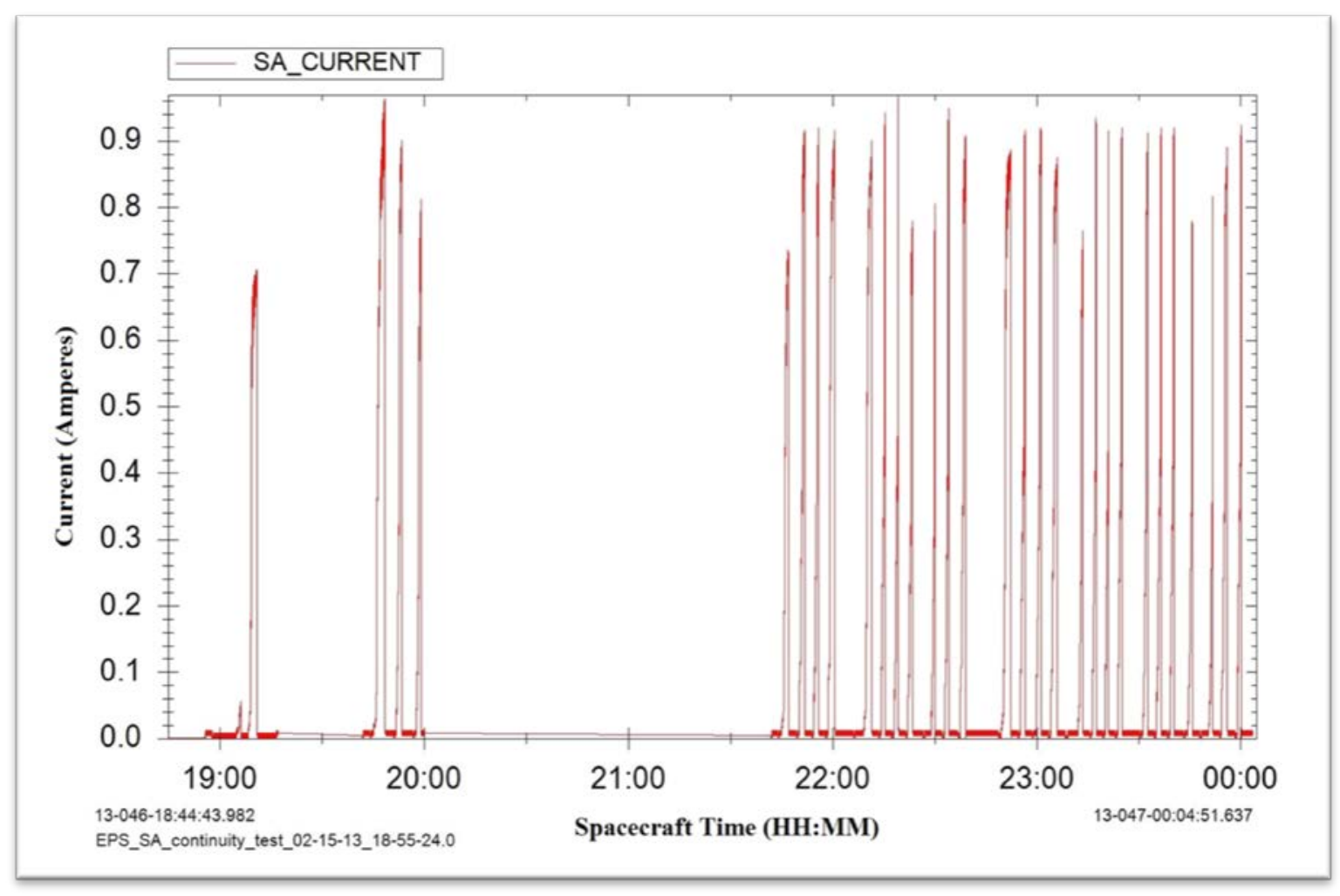

Figure 9: Solar Array Test Currents 


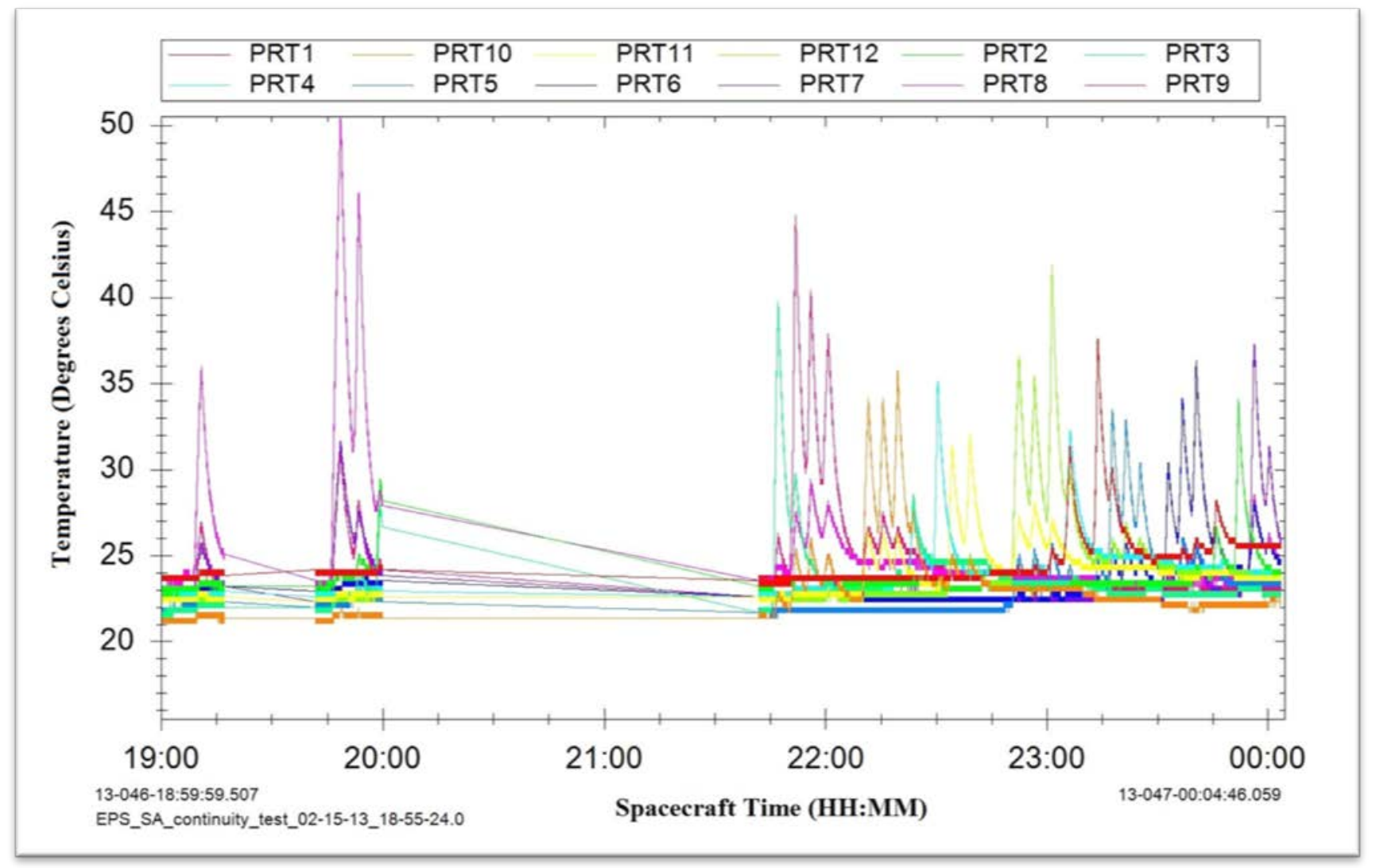

Figure 10: Solar Array Test Temperatures

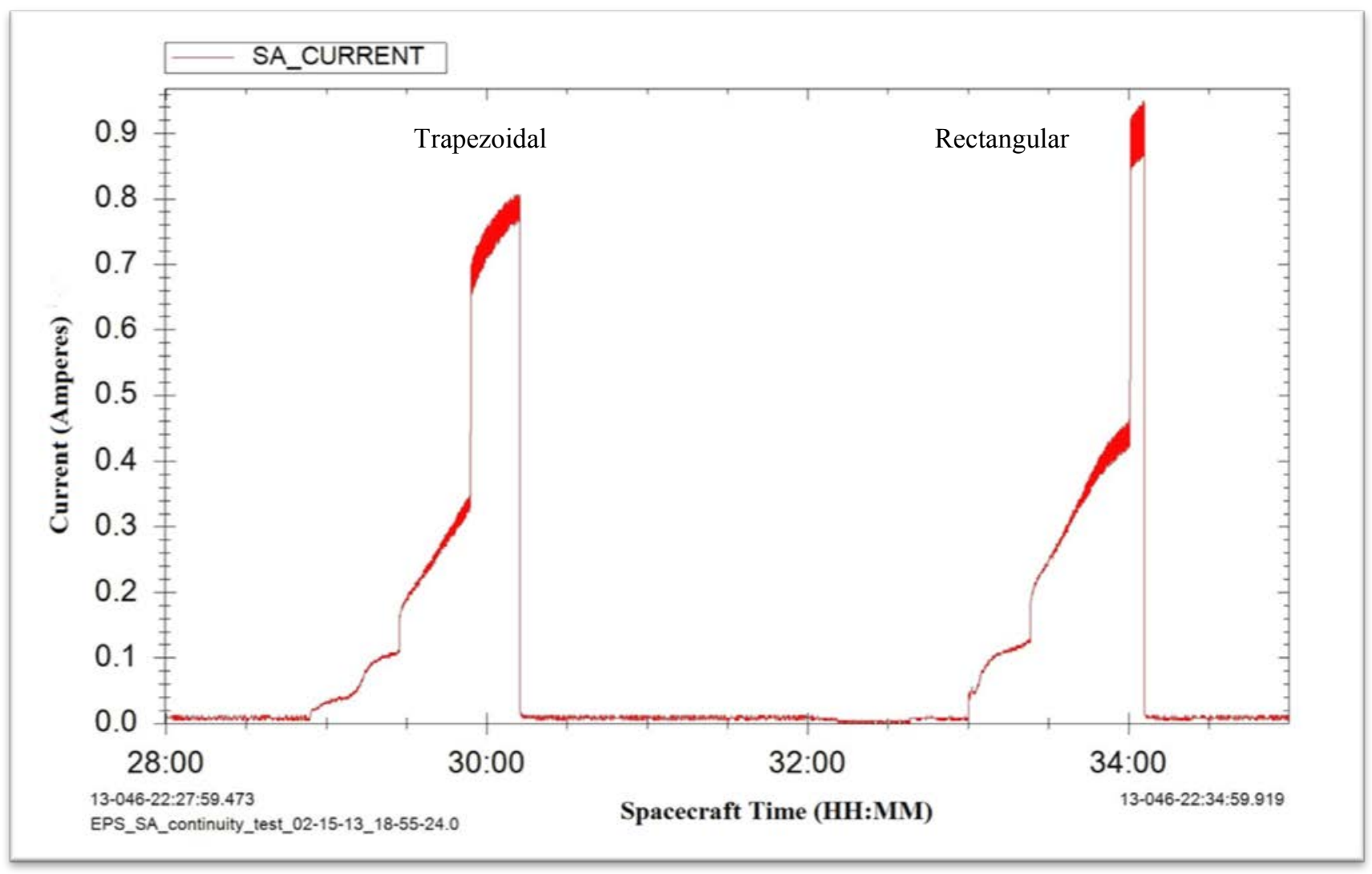

Figure 11: Typical Current Signature 


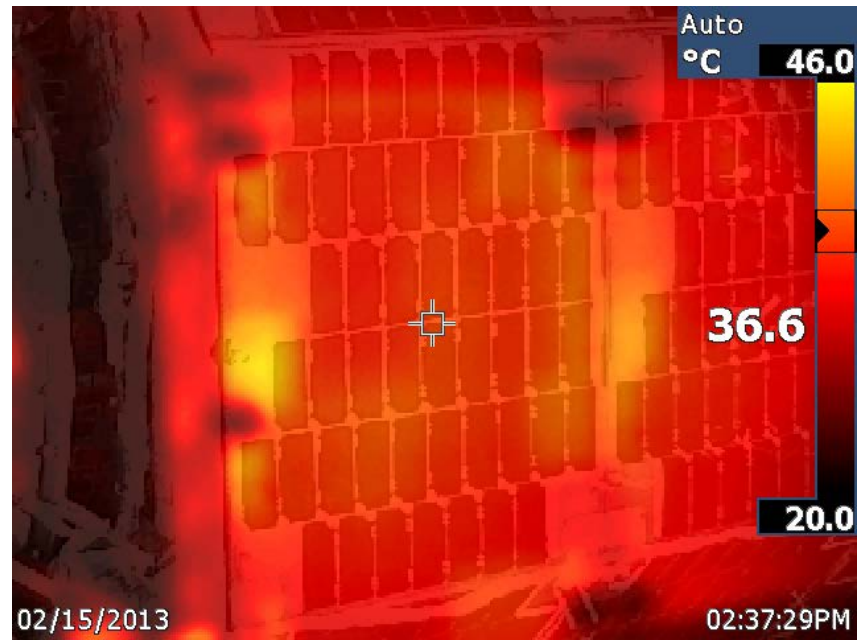

Figure 12: Infrared Image of a Panel during Test

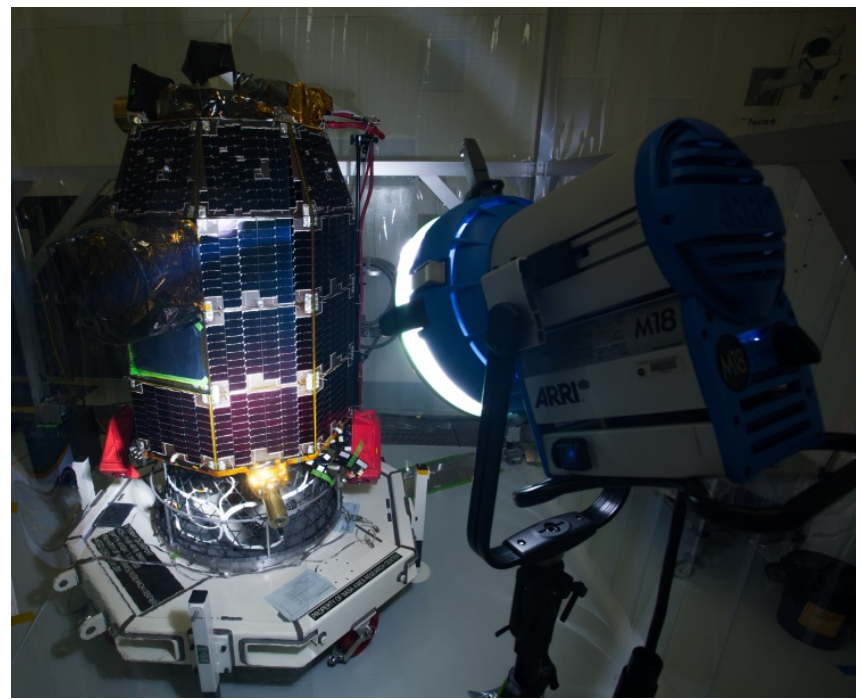

Figure 13: Final LADEE Test

\section{MAINTENANCE, INSPECTION, AND REPAIR}

\section{Receiving Inspection}

Beyond testing, the LADEE team used tried and true methods of visual inspection and record keeping from receipt through launch. The panel vendor accompanied the initial delivery of hardware to NASA ARC and participated in an on-site inspection, thereby documenting the initial state of the hardware. Throughout during Integration and Test (I\&T), the LADEE test team performed their own visual inspections of the hardware. This best practice resulted in the discovery of two panels with minor damage.

\section{Launch-Site Inspection}

Following shipment to the launch facility (NASA Wallops Flight Facility, Virginia), the manufacturer performed an on-site inspection and repair of the panels. Roughly $15 \%$ of the array was found to have minor cracks and damage due to handling (Figure 14). During an extended stay at Wallops
Island, the repair team was able to fix all affected panels with no issues (Figure 15). The simplicity, portability, and ease of operation of the lamp test fixture made it easy to reevaluate all repaired panels prior to launch. Note the inspections and final test were not part of the project's original (observatory-LAPSS test) plan.

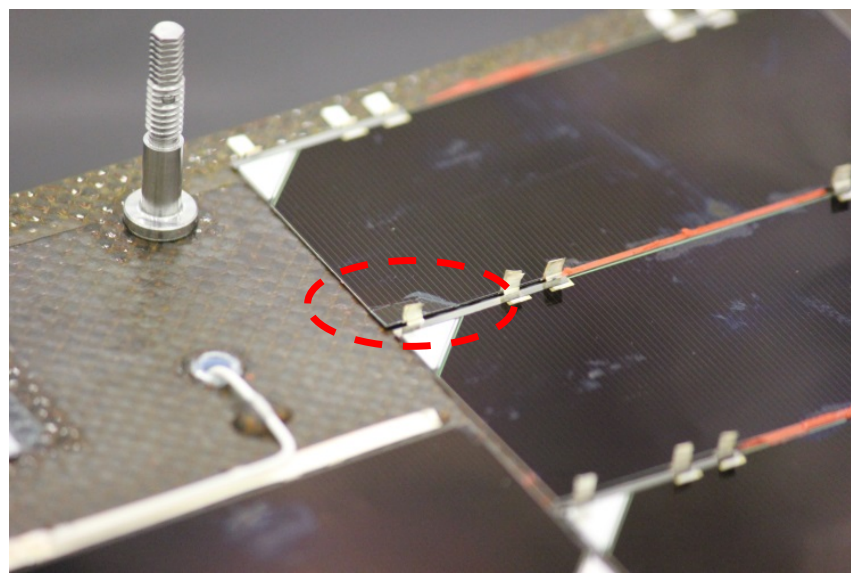

Figure 14: Damaged LADEE Solar Cell

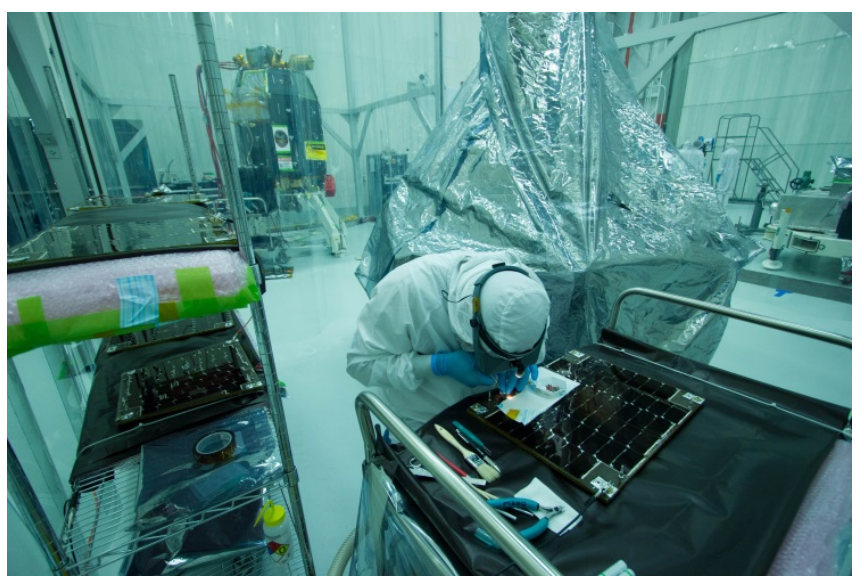

Figure 15: Cell Repair at the Launch Facility

\section{MISSION OPERATIONS}

A major assumption that enabled the entire risk mitigation process was the allocation of manufacturer's per-panel LAPSS data for requirements verification, LADEE EPS modeling and mission operations tools. Several factors can invalidate that assumption. One, obviously, is any damage and repair that could occur prior to launch. Another is degradation; though LADEE is a short-lifetime mission, some level of radiation or micro-meteorite damage will occur. A final factor, shadowing, is less a function of the panels themselves than the spacecraft geometry. Protrusions in the spacecraft will tend to shadow portions of the array, and predicting that behavior can be extremely complex. Neither the lamp test nor a LAPSS would completely account for shadowing. All of these factors feed into the need to continuously evaluate the health and performance of the solar array through mission operations. 
This would be relatively simple if the spacecraft's COTS avionics measured current from each panel, or even each switch. Unfortunately this is not the case; only one measurement is made for the entire solar array system. This design approach, while appropriate for a lowcomplexity Class D system, is highly inconvenient for debugging any type of photovoltaic system failure.

During Operational Readiness Training (ORTs), the LADEE EPS team developed a method to evaluate panel performance throughout the mission. The approach illuminates the interdisciplinary nature and value of mission operations experience, even for the hardware designer. In this case, the ORT campaign engendered collaboration between the EPS and Guidance, Navigation, and Control (GNC) teams that led to a solution. The EPS team discovered that the GNC system design includes calculation and storage of the spacecraft sun vector as part of its attitude determination process. As mentioned, the EPS subsystem records the solar array current and number of closed switches. The EPS analysis tool simply combines the downlinked sun vector with the EPS data and a geometrical spacecraft model to determine an expected array current, and the derived error (Figure 16). Observation of the spacecraft over a combination of attitudes can be used to back out the behavior of individual panels, thereby updating the manufacturer's data. project decision driven by resources, combined with redefinition of risk metrics, forced its staff to reconsider standard practices and determine what was really necessary. The process of test research development, execution, and risk closure took roughly 10 weeks (Figure 5). The total cost was roughly $12 \%$ of the industry-standard (LAPSS) solution in materials costs, and 12 labor-hours per test. Further, the theatre-lamp approach is easy to train with and portable. When combined with standard visual inspection, it actually added value over the LAPSS approach in this application. The test proved the system met power generation requirements 6 weeks prior to launch. Postlaunch, interdisciplinary approaches proved useful to track performance of the array in flight. Finally, the entire approach can be easily handed down to future missions.

\section{ACKNOWLEDGEMENTS}

This work was performed under the direction of Joseph Camisa, who served as the LADEE EPS Lead until his retirement in mid-2013. Additional notes of appreciation go to the NASA GSFC Power Group (Code 563), the Emcore inspection and repair staff, and the LADEE Systems Engineering, Project Management, and Integration and Test teams for their participation in this work.

\section{Conclusions}

The LADEE program applied true Class D risk tracking and mitigation techniques to address a major technical risk. A

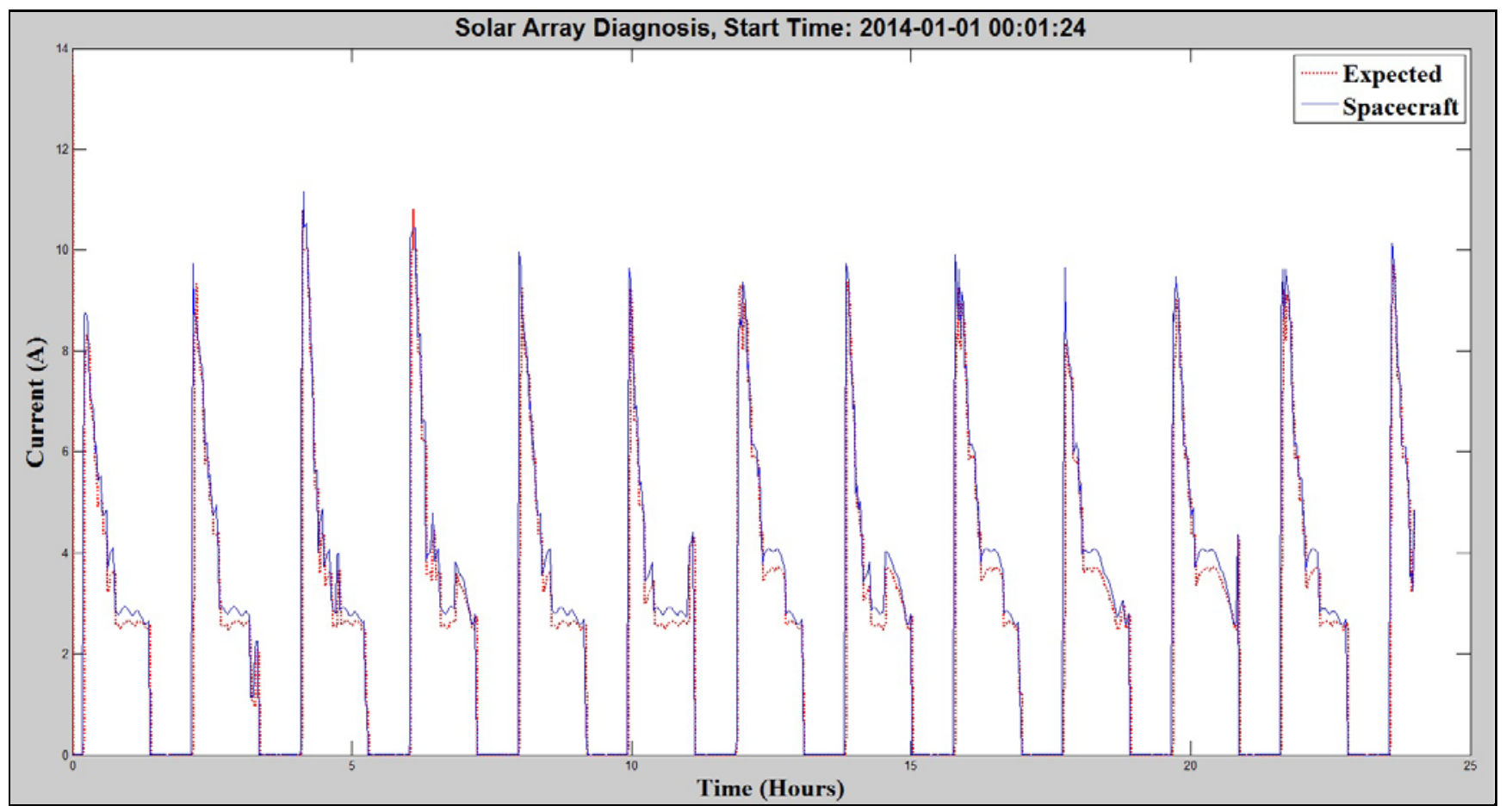

Figure 16 - Mission Operations Tool Overlays Expected and Actual Generated Array Current 


\section{REFERENCES}

[1] Hine, Butler et. al., "The Lunar Atmosphere and Dust Environment Explorer Mission," in Institute of Electrical and Electronics Engineers Aerospace Conference, Big Sky, MT, 2010, pp. 1-9.

[2] National Aeronautics and Space Administration, (1993, Aug. 15). The Large Area Pulse Solar Simulator (LAPSS) [Online]. Available: http://ntrs.nasa.gov.

[3] National Aeronautics and Space Administration, (2013, June 12). Risk Classification for NASA Payloads [Online]. NPR 8705.4, Change 2.

[4] Office of Procurement (2012, Aug. 10). Large Area Pulsed Solar Simulator - LAPSS - Testing [Online]. Available: http://fedbixopps.gov.

[5] Camisa, J. (2010, Aug. 12). Electrical and Power Systems Specification [Online]. SPEC-004.LADEE.EPSS, Revision NC, Internal use only.

[6] Emcore Photovoltaics (2012, June 15). End Item Data Package for LADEE [Online]. Internal use only.

[7] Forgione, J. (2013, Feb. 14). LADEE Solar Panel Continuity Test [Online]. I\&T-271.LADEE.SPCT Rev. A, Internal use only.

[8] Hudson Jr., Richard D., "Infrared Radiation," in Infrared System Engineering, $1^{\text {st }}$ ed., New York: Wiley, 1969, ch. 2, pp. 35-36.

[9] Biebl, B., et. al. (2009, Sept 8). ARRI M18 Spectral Data [Online]. Email to author.

\section{BIOGRAPHY}

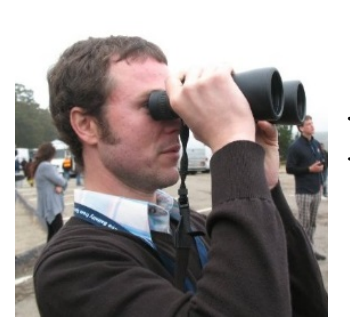

Josh Forgione received the B.S. degree in Electrical Engineering from Virginia Tech in 2000. He joined NASA in 2002, first at Goddard Spaceflight Center (GSFC) and then Ames Research Center in 2007. Josh's hardware deliverables are currently flying on the Aquarius/SAC-D satellite, the NASA ER-2, Global Hawk, and WB-57 aircraft, and multiple airborne instruments. He joined LADEE in 2011 and currently serves as the LADEE Electrical Power System lead through mission operations. His interests span electronics and electrical systems, particularly on analog design for scientific instruments and power systems.

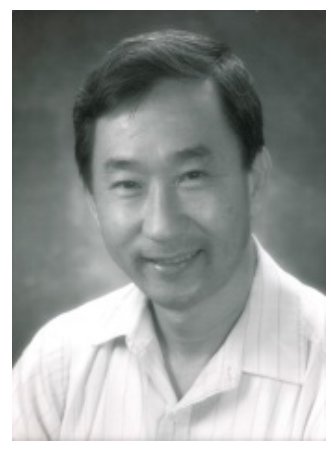

Gilbert Kojima was born in Honolulu, Hawaii, in 1949. He received the B.S and M.S. degrees in electrical engineering from the University of Hawaii, Honolulu, HI, in 1971 and 1974, respectively. He is began his career at NASA, Ames Research Center (ARC), Moffett Field, CA, as a physicist, then as an electrical/electronics engineer.

During his career at ARC, he has designed and developed numerous electronic instruments and major electrical control systems to support the diverse array of research and development activities at NASA. One of his major career accomplishments was as the principal tunnel conditions controls engineer for Unitary Modernization Project. His interest includes anything new and challenging involving electrical/electronic design.

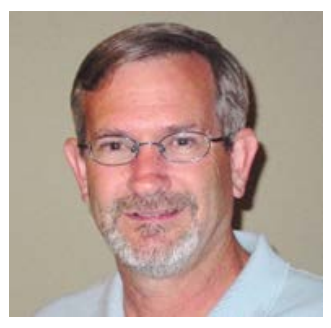

Robert Hanel is a Systems Engineer at NASA Ames Research Center currently working on the Lunar Atmospheric Dust Environment Explorer (LADEE) Mission. Robert has 25 years of experience working instruments, spacecraft systems, and mission designs for NASA scientific mission including work on Planetary, Astrophysics, and Space Biology Missions. Robert has a M.S. and a B.S in Electrical Engineering from California Polytechnic State University in San Luis Obispo.

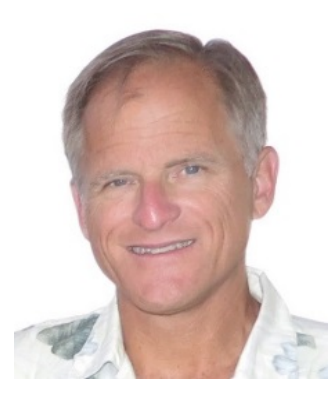

Mark Mallinson began working at NASA Ames Research Center in 1988 and holds Bachelor's and Master's degrees in Mechanical Engineering. Professional accomplishments include work on seven Space Shuttle missions, three satellites, wind tunnels, numerous aircraft and humanrated test facilities. Areas of NASA experience include structural modeling, test verification and analysis, systems design, project management and systems engineering at the program and project levels. Mark has held a number of systems engineering positions on the LADEE project including Risk Management Officer and manager of the project's Joint Confidence Level (JCL) cost/risk/schedule statistical analysis. 